\title{
RECENT DEVELOPMENTS IN SCANNING TUNNELING MICROSCOPY
}

\author{
R. WIESENDANGER \\ Institute of Applied Physics and Centre for Microstructural Research \\ University of Hamburg \\ Jungiusstrasse 11, 2000 Hamburg 36, Germany
}

\begin{abstract}
Scanning tunneling microscopy and related local probe methods have led to a novel perception of nanometer- and atomic-scale structures and processes. Since the structural information is obtained directly in real space, the scanning probe techniques offer considerable advantages compared with diffraction techniques for the investigation of non-periodic structures at solid surfaces. In addition, the local probe methods allow to study almost any kind of physical property of microstructures with submicron down to atomic resolution.
\end{abstract}

PACS numbers: $61.16 . \mathrm{Bg}, 68.35 . \mathrm{Bs}, 75.25 . \mathrm{tz}$

\section{Introduction}

Scanning tunneling microscopy (STM)/spectroscopy (STS) has become the most powerful tool to study the atomic-scale surface structure of electrically conducting solid surfaces [1-4]. Combined STM/STS studies provide valuable information about the relationship between surface atomic and local electronic structure, particularly for semiconductor surfaces $[5,6]$. The surfaces of poorly conducting substrates or bulk insulators can be investigated by force microscopy/spectroscopy $[7,3,4]$ yielding information about the atomic-scale topography as well as local mechanical properties. By using a magnetic tip in force microscopy, spatial variations of the stray field at surfaces of magnetic substrates can be probed. More recent developments in STM/STS aimed at the demonstration of chemical [8] as well as magnetic contrast [9] at the atomic level. These additional capabilities of STM/STS are important for the investigation of multicomponent and magnetic materials, respectively, thereby extending the useful applicability of STM/STS to more complex systems. 


\section{Atomic-scale structure}

Atomic-resolution topographs can easily be obtained by STM applied to semiconductor surfaces with dangling bonds. STS even allows to map out the spatial variation of different types of dangling bonds showing up at different energies. Combined with other surface analytical techniques, STM/STS has allowed to solve numerous structures of reconstructed surfaces during the past ten years $[2,5,6]$. In addition, non-periodic surface structures such as point defects, steps, dislocations, and grain boundaries can be characterized directly in real space [10]. This is even possible in the presence of relatively thick native oxide layers if force microscopy is used to measure the surface topography [11].

\section{Chemical contrast}

The distinction between different chemical species by STM/STS has been achieved in a few favorable cases only. For compound semiconductors, such as GaAs, for which the direction of charge transfer between the two types of atomic sites is known, bias-polarity dependent STM studies can offer atom-selective images of the two different species [12]. Besides spectroscopic STM/STS studies, simultaneous laser excitation has recently been proposed to achieve chemical contrast down to the atomic level [8]. In particular, the use of coordinated electronic and photon biasing has been demonstrated to provide specific atomic imaging for $\mathrm{CuInSe}_{2}$, a covalently bonded semiconductor with applications for thin-film photovoltaic technologies. It is assumed that the photon bias at a particular wavelength directly excites the atom, with the wavelength corresponding to the atomic transition probability. This method has been applied to study the local chemical order in the vicinity of defects such as grain boundaries.

\section{Magnetic contrast}

Magnetic contrast is achieved, for instance, by magnetic force microscopy (MFM) probing the surface magnetic stray field distribution [13, 14]. The long-range nature of the magnetic dipole interaction limits the spatial resolution achieved in MFM images to about 10-20 nm. By exploiting the spin dependence of the tunneling current flowing between two magnetic electrodes, a spin-polarized STM has been developed having the potential of providing magnetic contrast down to the atomic level. Vacuum tunneling of spin-polarized electrons in STM was first demonstrated by probing the alternately magnetized terraces of a $\mathrm{Cr}(001)$ surface by means of a ferromagnetic $\mathrm{CrO}_{2}$ tip [15]. Subsequent improvements in the in situ preparation of atomically sharp and chemically well-defined ferromagnetic STM probe tips.[16, 17] finally led to the first observation of magnetic contrast on the atomic scale $[9,18,19]$. This was demonstrated by using a $\mathrm{Fe}_{3} \mathrm{O}_{4}(001)$ surface for which the two different magnetic ions, $\mathrm{Fe}^{2+}$ and $\mathrm{Fe}^{3+}$, on the octahedrally coordinated iron sites of magnetite were distinguished by a ferromagnetic iron tip. The contrast mechanism is most likely due to spin-polarized tunneling, whereas long-range magnetic interactions cannot lead to an atomic-scale contrast. 


\section{Summary}

STM and related local probe methods allow to study atomic-scale surface structures and their physical properties directly in real space. Recent developments in STM/STS have led to first demonstrations of chemical as well as magnetic contrast at the atomic level which further extends the applicability of STM/STS. In particular, the investigation of the relationship between chemical, magnetic, mechanical properties and the underlying atomic-scale structure, as one of the major challenges in the research on microstructures, can now be performed in an even more powerful way.

\section{References}

[1] G. Binnig, H. Rohrer, Rev. Mod. Phys. 59, 615 (1987).

[2] Scanning Tunneling Microscopy I, Springer Series in Surface Sciences, Vol. 20, Eds. H.-J. Güntherodt, R. Wiesendanger, Springer, Berlin 1992.

[3] Scanning Tunneling Microscopy II, Springer Series in Surface Sciences, Vol. 28, Eds. R. Wiesendanger, H.-J. Güntherodt, Springer, Berlin 1992.

[4] Scanning Tunneling Microscopy III, Springer Series in Surface Sciences, Vol. 29, Eds. R. Wiesendanger, II.-J. Güntherodt, Springer, Berlin 1993.

[5] R.J. Hamers, Ann. Rev. Phys. Chem. 40, 531 (1989).

[6] R.M. Feenstra, in: Scanning Tunneling Microscopy and Related Methods, Eds. R.J. Behm, N. Garcia, H. Rohrer, NATO ASI Series E: Appl. Sci., Vol. 184, Klüwer, Dordrecht 1990, p. 211.

[7] G. Binnig, C.F. Quate, Ch. Gerber, Phys. Rev. Lett. 56, 930 (1986).

[8] L.L. Kazmersky, J. Vac. Sci. Technol. B 9, 1549 (1991).

[9] R. Wiesendanger, I.V. Shvets, D. Bürgler, G. Tarrach, II.-J. Güntherodt, J.M.D. Coey, Europhys. Lett. 19, 141 (1991).

[10] R. Wiesendanger, G. Tarrach, D. Bürgler, L. Scandella, H.-J. Güntherodt, Mater. Res. Soc. Symp. Proc. 183, 237 (1990).

[11] H. Bluhm, R. Wiesendanger, B. Keller, U.D. Schwarz, P. Paufler, not published.

[12] R.M. Feenstra, J.A. Stroscio, J. Tersoff, A.P. Fein, Phys. Rev. Lett. 58, 1192 (1987).

[13] Y. Martin, H.K. Wickramasinghe, Appl. Phys. Lett. 50, 1455 (1987).

[14] J.J. Saenz, N. Garcia, P. Grütter, E. Meyer, H. Heinzelmann, R. Wiesendanger, L. Rosenthaler, H.R. Hidber, H.-J. Güntherodt, J. Appl. Phys. 62, 4293 (1987).

[15] R. Wiesendanger, H.-J. Güntherodt, G. Düntherodt, R.J. Gambino, R. Ruf, Phys. Rev. Lett. 65, 247 (1990).

[16] R. Wiesendanger, D. Bürgler, G. Tarrach, T. Schaub, U. Hartmann, H.-J. Güntherodt, I.V. Shvets, J.M.D. Coey, Appl. Phys. A 53, 349 (1991).

[17] R. Wiesendanger, D. Bürgler, G. Tarrach, I.V. Shvets, H.-J. Güntherodt, Mater. Res. Soc. Symp. Proc. 231, 37 (1992).

[18] R. Wiesendanger, I.V. Shvets, D. Bürgler, G. Tarrach, II.-J. Güntherodt, J.M.D. Coey, Z. Phys. B 86, 1 (1992).

[19] R. Wiesendanger, I.V. Shvets, D. Bürgler, G. Tarrach, H.-J. Güntherodt, J.M.D. Coey, S. Gräser, Science 255, 583 (1992). 